\title{
LES ULTIMES FEUX DE LA RÉSISTANCE PAÏENNE DANS L'ANTIQUITÉ TARDIVE
}

\author{
Stéphane Ratti \\ Université de Bourgogne - France
}

\section{Resumo}

Les travaux récents de l'auteur invitent à un examen renouvelé de la signification idéologique et religieuse de certaines des sources historiographiques majeures de la fin du quatrième siècle parmi lesquelles l'Histoire Auguste. Il apparaît en effet désormais que cette œuvre s'inscrit, avec d'autres, dans le contexte de l'ultime combat mené contre le christianisme triomphant par les derniers défenseurs de l'ancienne religion dont Nicomaque Flavien senior apparaît comme le chef de file. De 384, date de la mort de Prétextat, à 395, date de celle de Théodose, les païens ont pu espérer sauver les cultes anciens et ont même pris les armes en grande partie pour ce motif au Frigidus. Entre 395 à 408, soit jusqu'à la disparition de Stilichon, la lutte entre païens et chrétiens n'a pas cessé, mais sous une autre forme, les protagonistes des deux camps privilégiant alors l'arme littéraire. Les rééditions des auteurs classiques qui se multiplient alors constituent un élément essentiel de la lutte menée autour des Symmachi contre la christianisme et ne sont pas un simple divertissement littéraire d’intellectuels dés œuvrés. L'analyse conforte ainsi l'interprétation polémique au détriment de la vision irénique des relations pagano-chrétiennes à la fin du quatrième siècle.

\section{Resumo}

Os trabalhos recentes do autor convidam a um exame renovado do significado ideológico e religioso de certas fontes historiográficas importantes do final do século IV, entre as quais a História Augusta. De fato, parece que esta obra se inscreve, com outras, no contexto do último combate conduzido contra o cristianismo triunfante pelos derradeiros defensores da antiga religião da qual Nicômaco Flaviano sênior surge como o principal líder. De 384, data da morte de Pretextato, a 395, data da morte de Teodósio, os pagãos puderam esperar preservar os cultos antigos, chegando inclusive a armar-se em boa medida por este motivo, na batalha de Frígido. Entre 395 e 408, isto é, até o desaparecimento de Estilicão, a luta entre pagãos e cristãos foi ininterrupta, mas sob outra forma, os protagonistas dos dois campos privilegiando então a arma literária. As reedições de autores clássicos que se multiplicam então constituem um elemento essencial da luta conduzida em torno dos Symmachi contra o cristianismo e não se reduzem a um simples divertimento literário de intelectuais desocupados. A análise confirma assim a interpretação polêmica em detrimento da visão irênica das relações pagão-cristãs, no final do quarto século. 


\section{Introduction}

Lorsque les chrétiens, à la fin du quatrième siècle, rencontraient dans les champs une pierre ceinte de rubans qui, visiblement, servait au culte des anciens dieux païens, ils la brisaient rageusement. De même ils abattaient à coup de hache vengeresse l'arbre orné de bandelettes qui donnait lieu à l'expression du culte de la vieille religion. ${ }^{1}$ C'est Prudence qui le raconte dans le Contre Symmaque. Ce témoignage prouve qu'en 402-403, date du second livre de ce poème polémique antipaïen, ${ }^{2}$ les pratiques polythéistes n'avaient pas disparu des campagnes. Il illustre encore parfaitement la virulence du conflit pagano-chrétien à cette date relativement tardive, soit une dizaine d'années après le moment auquel la plupart des historiens placent la victoire définitive du christianisme, installé comme religion officielle de l'Empire romain grâce aux mesures législatives prises par Théodose entre 392 et 394. Les mots par lesquels Prudence choisit de qualifier le paganisme - moins moribond qu'il ne veut, avec ses coreligionnaires, bien le dire - sont ceux d'une “vieille lubie”, Antiquus error.

Ce sont ces termes que j'ai choisis de reprendre dans le titre de mon ouvrage qui paraît en 2009: Antiquus error. Les ultimes feux de la résistance païenne. ${ }^{3}$ L'objet de ce livre est de proposer, à la suite de plusieurs découvertes que j'ai pu faire entre 2004 et 2008, une thèse générale que je résumerai comme suit. La défaite militaire du Frigidus, ou bataille de la Rivière Froide, qui voit les 5 et 6 septembre 394 le camp païen s'incliner face aux troupe du très chrétien Théodose ne marque pas pour autant l'abdication définitive de la résistance païenne face au maître de l'Empire et à la nouvelle religion. Certes l'usurpateur Eugène, mais surtout Arbogast et Nicomaque Flavien senior - le véritable chef de file du camp païen - sont morts, mais la lutte prend désormais d'autres formes. Nicomaque Flavien senior était aussi un historien réputé ${ }^{4}$ et il a laissé avec l'Histoire Auguste - qu'il faut identifier avec les Annales perdues signalées par CIL 1783 - un brûlot antithédosien en même temps qu'un manifeste politique qui appelle à la tolérance en faveur de l'ancienne religion. Il avait même rédigé quelques années plus tôt une declamatio (la $3^{\text {ème }}$ déclamation pseudo-quinti-

\footnotetext{
${ }^{1}$ C. Symm. 2, 1006-1009: ... et lapis illic / si stetit, antiquus quem cingere sueuerat error / fasceolis uel gallinae pulmone rogare, / frangitur (...) / Et quae fumificas arbor uittata lucernas / Seruabat, cadit ultrici succisa bipenni.

${ }^{2}$ CHARLET, J.-L. Prudence. Psychomachie. Contre Symmaque. Paris: CUF, 1992, p. 89-91.

3 "Bibliothèque de l'Antiquité Tardive". Turnhout: Brepols, 2009, préface par J.-M. Carrié.

${ }^{4}$ Historicus disertissimus (CIL 6, 1782).
} 
lienne) de nature plus polémique encore, le Miles Marianus. Cette forme de lutte menée non plus sur le champ de bataille mais sur le terrain littéraire s’inscrit dans un mouvement plus large qui voit de nombreuses rééditions d'auteurs païens conduites avec un objectif idéologique de nature comparable dans les cercles érudits païens. C'est à une réévaluation de cette forme de résistance contre les idées dominantes aujourd’hui minorée par une certaine école historique que s’attache mon ouvrage dont je résume dans ce qui suit les lignes de force.

\section{Les rééditions des auteurs classiques dans l'Antiquité Tardive}

La diffusion des manuscrits s'était, dès le Haut-Empire, accompagnée d'une révision du texte, ce qu'on appelle l'emendatio. L'habitude se prit alors de signer le travail. Les copistes et réviseurs ajoutaient in fine leur nom, précisaient la nature de leurs interventions (legi; descripsi; emendaui), indiquaient parfois quel manuscrit leur avait servi de référence (ad exemplar; de codice) quand ils avaient la chance d'en disposer (on trouve aussi la formule sine exemplario; sine antigrapho) et localisaient leur intervention (Romae; apud Hennam; apud magistrum meum). Ces indications éminemment précieuses ont été elles-mêmes recopiées dans les manuscrits d'une même branche, ce qui est un élément clef pour l'établissement des stemmata. On a pris l'habitude de les appeler les subscriptiones. ${ }^{5}$ J'entends ainsi par “réédition” la mise en circulation d'un livre ou d'une œuvre complète d'un auteur ancien après révision, étant entendu que ces textes s'accompagnent d'une ou de plusieurs signatures, d'une localisation et d'une datation. Naturellement d'autres œuvres antiques que celles qu'attestent les subscriptiones circulaient. Mais même si elles étaient recopiées par des scribes dans des ateliers ou dans de grandes familles, je ne considère pas qu'elles méritent le label de "rééditions”. Ausone, par exemple, avait fait copier les Apologues de Titianus (troisième siècle?) et les Chronica de Cornelius Nepos pour le préfet du prétoire Sextus Anicius Petronius Probus, mais rien n'indique en l'occurrence un quelconque travail de révision. ${ }^{6}$

\footnotetext{
${ }^{5}$ L'étude de référence que laquelle je m'appuie et qui n'a jamais été remplacée est celle de Über die Subskriptionen in den Handschriften römischer Klassiker, Berichte der sächsischen Gesellschaft der Wissenschaft zu Leipzig, Phil.-Hist. Klasse 3, 1851, p. 327-372. L'étude porte sur 27 cas au total, commodément numérotés, mais enregistrés sans classement ni chronologique ni thématique. La distinction entre subscriptiones païennes et chrétiennes n'y est pas faite non plus. En outre O. Jahn a produit un travail de nature philologique - une édition des subscriptiones - et non un essai d'interprétation. ${ }^{6}$ Cf. AUSONE, epist. 16, 1, p. 174 Schenkl: Oblata per antiquarios mora, scio promissi mei
} 
La plus ancienne de ces révisions-rééditions au sens plein du terme remonte probablement au premier ou au second siècle, sans que l'on puisse préciser davantage: il s'agit de l'intervention de Statilius Maximus enregistrée dans une subscriptio au second discours de Cicéron De lege agraria prononcé contre Rullus. Ce cas, dans son ancienneté, paraît néanmoins isolé. La plus récente, quant à elle, figure dans un manuscrit d'Horace, révisé par Vettius Agorius Basilius, à Rome, en 527. L'arc chronologique couvre donc une vaste période.

Une forte concentration des subscriptiones doit être notée pour les $4^{\mathrm{e}}$ et $5^{\mathrm{e}}$ siècles. Pour être plus précis encore, nos témoignages s'inscrivent presque tous entre 380 et 450, soit entre la révision des Grandes déclamations du pseudoQuintilien ${ }^{7}$, sur laquelle je reviendrai, et la réédition de l'Epitoma rei militaris de Végèce, à Constantinople, par Flavius Eutropius, qui est parfaitement datée. ${ }^{8}$ La période correspond donc assez exactement aux règnes de Théodose I et de son petit-fils Théodose II.

Néanmoins une distinction d'importance doit être opérée, celle qui isole les subscriptiones émanant de chrétiens connus par ailleurs comme tels ou dont la signature, accompagnée de formules telles que Christo adiuuante, est sans équivoque. Dès lors deux groupes peuvent être déterminés dont la ligne de partage passe par l'année 408. Avant cette date, et si l'on excepte le cas atypique et impossible à dater de Cicéron, on enregistre six noms d'auteurs classiques réédités: Apulée (Métamorphoses; Apologie); Martial; Virgile; Perse; Tite-Live (1 $1^{\text {èr }}$ décade) et Juvénal. Seule la réédition de Juvénal par Niceus ne peut formellement être datée, mais la mention apud Seruium renvoie évidemment au commentateur de Virgile, Maurus Honoratus Servius, et situe l'activité de Niceus à la fin du quatrième siècle. Les autres dates sont assurées: Apulée est réédité deux fois, la première en 395, la seconde en 397, Martial l'est en 401, ${ }^{9}$ Perse en 402, les dix

gratiam exspectatione consumptam, Probe, uir optime. In secundis tamen habeo non fefelisse. Apologos Titiani, et Nepotis Chronica, quasi alios apologos (nam et ipsa instar sunt fabularum) ad nobilitatem tuam misi, gaudens atque etiam glorians fore aliquid, quod ad institutionem tuorum, sedulitatis meae studio, conferatur.

${ }^{7}$ L'étude d'O. JAHN (citée n. 5) ne mentionne pas les declamationes falso Quintiliano adscriptae.

${ }^{8}$ Fl. Eutropius emendaui sine exemplario Constantinopolim consul. Valentiniano Aug. VII et Abieni, p. 165 Lang.

${ }^{9}$ O. JAHN (cité n. 5), p. 331, plaçait à tort Gennadius Torquatus sous Constantin. La date de 401 a été retenue par LOMMATZSCH, E. Litterarische Bewegungen in Rom im vierten und fünften Jahrhundert n. Chr., Zeitschrift für vergleichende Litteraturgeschichte N. F. 15, 1904, p. 177-192, ici p. 186, par LABRIOLLE, P. de. La réaction païenne. Étude sur la polémique antichrétienne du I ${ }^{\mathrm{er}}$ au VI ${ }^{\mathrm{e}}$ siècle. Paris, 1934, rééd. 2005, p. 355, et par la PLRE II, p. 1124, s. u. Gennadius Torquatus. 
premiers livres de Tite-Live entre 401 et 408. Quant à la réédition sous la forme d'un recueil des declamationes maiores du pseudo-Quintilien, elle est de peu antérieure à $384 .{ }^{10}$ Sept auteurs, pour résumer, font l'objet d'une révision-réédition entre 384 et 408 , et six d'entre eux plus précisément entre 395 et 408 . Cette concentration remarquable porte en elle sa signification: de 384, date de la mort de Prétextat, à 395, date de celle de Théodose, les païens ont pu espérer sauver les cultes anciens et ont même pris les armes en grande partie pour ce motif au Frigidus; entre 395 et 408, soit jusqu'à la disparition de Stilichon, la lutte entre païens et chrétiens n'a pas cessé, mais s'est développée sous une autre forme, les protagonistes des deux camps privilégiant alors l'arme littéraire. Je rappelle pour mémoire qu'alors, côté païen, est déjà parue l'Histoire Auguste et l'intégralité des Res Gestae d'Ammien Marcellin, et côté chrétien, le commentaire In Danielem de Jérôme ainsi que la trilogie des pamphlets antipaïens anonymes, le Carmen contra paganos, le Carmen ultimum et le Carmen ad senatorem.

Le second groupe des subscriptiones circonscrit une toute autre période, celle qui s'ouvre avec la disparition de Théodose II en 450 et qui va jusqu'à l'époque de la royauté ostrogothique de Théodoric. Cette seconde série est inaugurée par la réédition de Végèce par Flavius Eutropius, en 450. Elle se clôt par les révisions de Priscien et d'Horace qui circulent en Occident vers 526-527. Au total seize auteurs classiques ont alors été réédités, parmi lesquels Térence, Virgile, Pomponius Mela ou encore Pline l'Ancien.

Le tableau ci-dessous résume commodément les rééditions attestées par les suscriptions:

${ }^{10}$ Cf. SCHNEIDER, C. Quelques réflexions sur la date de publication des Grandes déclamations pseudo-quintiliennes. Latomus 59 (3), 2000, p. 614-632, ici p. 616-617. 


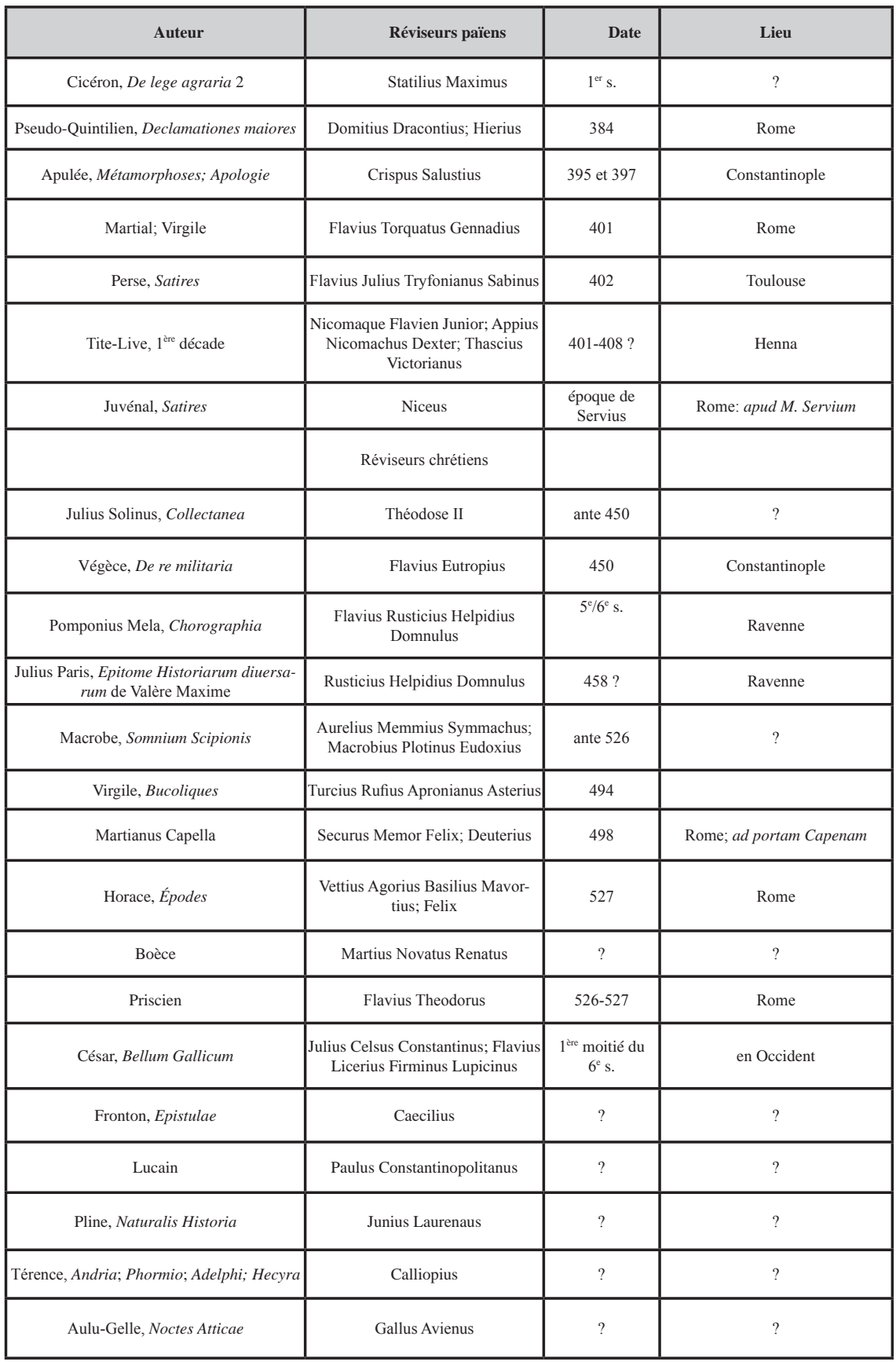




\section{Réaction païenne ou divertissement littéraire?}

Venons-en, après cet essai de description et de définition, à l'interprétation des subscriptiones d'origine païenne. La période circonscrite à l'instant, disons entre 384 et 408, correspond naturellement à la christianisation des pratiques, des institutions et des lois - sinon des âmes, ce qui est un autre problème. C'est même dans ce créneau que l'on situe traditionnellement la victoire définitive du christianisme sur les derniers païens, quelque part entre la polémique sur l'Autel de la Victoire qui a opposé Symmaque et Ambroise, la défaite d'Eugène au Frigidus en 394 et les lois religieuses des deux Théodoses. Il était donc naturel que la dimension religieuse conditionnât l'interprétation de la floraison des rééditions telle que les subscriptiones nous la font revivre. Afin de cadrer le débat et de mieux situer ma propre lecture que je donnerai plus loin je rappelle l'importance de quelques travaux modernes sur le sujet. Deux tendances très marquées et franchement opposées peuvent en effet être définies.

La première école est représentée par l'ouvrage classique, réédité à maintes reprises et encore tout récemment - ce qui atteste sa notoriété en France - de Pierre de Labriolle, La réaction païenne, paru initialement en 1934. Pour l'auteur il ne fait guère de doute que les rééditions en question s’inscrivent dans un courant idéologique et religieux de résistance active au christianisme. Les païens avaient choisi les seules armes encore en leur possession après la défaite de l'usurpateur Eugène: la lutte intellectuelle par œuvres littéraires interposées. Dans ce contexte, les rééditions cherchent à illustrer et démontrer la grandeur de la Rome des temps jadis, le prestige d'une civilisation païenne que l'on pense ainsi revivifier: "Il est difficile de ne pas imputer, pour une large mesure, au même souci de revigorer les traditions du passé, déjà ébranlées et vacillantes, l'ardeur avec laquelle, vers la fin du IV siècle et le début du V $V^{e}$ siècle, on voit des membres de l'aristocratie romaine, des lettrés, des rhéteurs, faire copier les manuscrits des écrivains classiques ou les réviser de leur mieux pour assurer la pureté du texte”. ${ }^{11}$ À cette même école se rattachent plusieurs travaux majeurs d'Andreas Alföldi ${ }^{12}$ et deux études d'Herbert Bloch, un historien dont les travaux

\footnotetext{
${ }^{11}$ LABRIOLLE, P. de. La réaction païenne. Étude sur la polémique antichrétienne du $\mathrm{I}^{\mathrm{er}}$ au VI siècle. Paris, 1934, rééd. 2005, p. 354.

${ }^{12} \mathrm{~A}$. ALFÖLDI a par exemple montré que, à la fin du $4^{\mathrm{e}}$ siècle, le thème de la culture du Prince était un objet de polémique politique mais jamais complètement dénué d'arrières plans religieux: cf. A conflict of ideas in the late Roman Empire. The clash between the Senate and Valentinian I. Oxford, 1952, p. 122-124.
} 
s'ils sont parfois cités ne sont pas toujours considérés à leur juste valeur. H. Bloch s'est notamment exprimé très fermement dans un article essentiel qui, en 1964, reprend, dans sa partie finale, l'examen des subscriptiones conservées pour conclure que leur interprétation ne saurait être détachée artificiellement du contexte religieux et affirmer qu'elles constituaient bien un élément essentiel de la lutte alors menée autour des Symmachi contre le christianisme. ${ }^{13}$

Dans l'autre camp, si je puis dire, on trouve l'école anglo-saxonne largement influencée par les travaux d'Alan Cameron. Un bon résumé de la position de ce dernier se trouve exposé dans un article de $1977 .{ }^{14}$ En gros, explique A. Cameron, on a surévalué l'importance des œuvres littéraires dans le conflit religieux, qui n'a, d'ailleurs, selon lui, rien eu de virulent. L'affaire de l'Autel de la Victoire? On a grossi son importance. ${ }^{15}$ Le conflit entre Eugène et Théodose? Ce fut un conflit politique pour le pouvoir dont la dimension religieuse n'était qu'un prétexte. ${ }^{16}$ Pour A. Cameron, les intellectuels païens n'ont jamais été que d'aimables antiquaires occupés, dans le secret et la douceur de leur cabinet de travail, à d'innocents travaux d'érudition sans aucune portée idéologique. Le meilleur exemple à ses yeux étant les Saturnales de Macrobe, un recueil de curiosa dont la petitesse voire l'insignifiance discrédite toute tentative de prêter à son auteur la moindre intention polémique. D'ailleurs la preuve définitive en est fournie par la date tardive (après 430) à laquelle A. Cameron croit pouvoir placer la rédaction des Saturnales, ${ }^{17}$ position discutable - et insuffisamment discutée - sur laquelle je reviendrai ailleurs. La thèse d'A. Cameron repose en fait sur deux piliers. Un: on ne peut prêter aucun sens aux rééditions des œuvres antiques entreprises par les Symmachi ou autour d'eux pour la raison simple qu'il n’y

\footnotetext{
${ }^{13}$ The Pagan Revival in the West at the End of the Fourth Century, dans MOMIGLIANO, A. The conflict between paganism and christianity in the fourth century. Oxford, 1964, p. 193-218; l'examen des subscriptiones est fait dans les pages 214-217; cf. aussi id., A new document in the last pagan revival in the West, 393-394 A. D., Harvard Theological Review 38, 1945, p. 199-244. ${ }^{14}$ CAMERON, A. Paganism and literature in late fourth century Rome, dans Christianisme et formes littéraires de l'Antiquité tardive en Occident, Entretiens sur l'Antiquité Classique, Fondation Hardt, Vandœuvres-Genève, 1977, p. 1-30.

${ }^{15}$ Paganism and Literature... (cité n. 14), p. 1, n. 1: "The importance of this affair has been exagerated in modern accounts of the Christianization of the Roman aristocracy".

${ }^{16} \mathrm{Cf}$. PASCHOUD, F. Pour un mille six centième anniversaire: le Frigidus en ébullition. Antiquité Tardive 5, 1997, p. 275-280, repris dans Olympiodore, Eunape, Zosime. Bari: Scripta minora, 2006, p. 353-364, avec une Retractatio importante, p. 364-366, qui répond par anticipation au livre encore inédit d'A. CAMERON, The last pagans of Rome.

${ }^{17}$ The date and identity of Macrobius, The Journal of Roman Studies 56, 1966, p. 25-38.
} 
eut jamais de "Cercle de Symmaque" ni, a fortiori, de projet cohérent construit autour d'eux, ni même de lien entre la plupart des rééditions et aucun membre de la gens des Symmaques ou des Flaviens. Deux: les auteurs païens ne lisaient pas les œuvres des chrétiens (alors que l'inverse n'est pas vrai); ils ne pouvaient donc pas leur répondre dans un affrontement de nature littéraire puisqu’ils en ignoraient les écrits. Je reviendrai dans un instant sur ces points décisifs.

Très récemment un livre publié aux États-Unis dont l'objet principal n'est pas tout à fait le nôtre ici exprime, dans plusieurs chapitres préliminaires, un point de vue qui touche néanmoins de près à l'interprétation des rééditions ${ }^{18}$. Il s'agit d'un ouvrage de nature historiographique qui ne répond pas exactement aux normes universitaires habituelles puisque son ambition relève, au fond, de l'épistémologie. Quoi qu'il soit écrit dans un anglais très pur et parfaitement compréhensible, il correspond plutôt à ce que nous appellerions un essai. L'auteur prend en effet pour point de départ l'inscription de 431 qui réhabilite la mémoire de Nicomaque Flavien senior ( $C I L 6,1783)$ pour hausser sa plume jusqu'à une réflexion sur la mémoire historique. En fait, l'affaire Nicomaque Flavien - sa damnatio memoriae - semble bien un prétexte ou une métaphore pour réfléchir sur l'oubli dans l'histoire, sur la manipulation des mémoires et même sur les tentations du pouvoir, quel que soit son époque, d'écrire une histoire officielle, faite d'érasures et d'effacements des vérités dans les consciences ${ }^{19}$. Le projet est très intéressant et l'idée féconde, mais, on l'aura compris, l'auteur n'étant pas un spécialiste du quatrième siècle, mais une espèce de sociologue qui emprunte ses références tantôt à la littérature sociale américaine contemporaine tantôt à un Lacan fort simplifié ${ }^{20}$ et qui prend l'Antiquité comme objet, son interprétation

\footnotetext{
${ }^{18}$ HEDRICK JR., C. W. History and silence: The purge and rehabilitation of memory in late Antiquity. Austin: University of Texas Press, 2000.

${ }^{19}$ L'auteur me paraît marqué, dans sa réflexion sur les “minorités", par le politiquement correct: cf. p. 52; cf. note 55 , p. 268 , avec une bibliographie curieuse. Fallait-il tout ce développement pour en arriver à la conclusion que le paganisme ne se définit que par opposition au christianisme, ce qui est en soi parfaitement contestable (il n’y aurait ainsi pas de paganisme conscient avant les chrétiens, au moins sous un autre nom?) et qui, de toute façon, me semble plaider plutôt pour la prise en compte de la dimension polémique, ce que les pages précédentes remettent pourtant en cause (e. g. p. 47). - Et puis, si l'on entre dans le raisonnement de l'auteur, il devient immédiatement perceptible qu'il conduit à la contradiction suivante: si le paganisme a besoin d'un repoussoir pour exister, c'est donc que la relation dans ce couple est bien de l'ordre de la construction par l'opposition ou la symétrie; la neutralité ou la coexistence indifférente n'est en fin de compte pas dans la nature de leurs relations, ce qui est exactement l'inverse de la thèse que veut démontrer C. W. Hedrick. ${ }^{20}$ Cf. p. 268 , note 54 .
} 
de la période 350-430 me paraît sujette à caution. Je m'attarde un instant sur ce livre non pas seulement en raison de la parution d'un compte rendu curieusement dithyrambique, ${ }^{21}$ mais parce qu'il me paraît représenter un cas d'école. Dans son chapitre 3 (p. 37-88), l'auteur, en effet, passe en revue les interprétations opposées que donnent H. Bloch et A. Cameron des événements de 380-394 afin d'éclairer le sens de la réhabilitation de 431. L'enquête révèle une bonne connaissance des arguments de l'un et de l'autre mais se solde par une conclusion fort décevante ou plutôt par une absence de conclusion: après avoir renvoyé dos à dos les deux écoles, C. W. Hedrick se réfugie dans des considérations méthodologiques et abstraites sur le rôle sélectif de la mémoire sans proposer rien de bien personnel concernant mon sujet. Voilà où conduit, me semble-t-il, la position neutraliste défendue par $\mathrm{A}$. Cameron, qui ne doit sans doute guère se réjouir d'avoir été ainsi annexé par l'école sociologique américaine: les faits seraient susceptibles d'être interprétés dans un sens ou dans un autre. Car enfin, en affirmant que les intellectuels tout en célébrant la grandeur de la Rome passée ne polémiquaient pas avec les chrétiens, on dénie que la période ait été celle d'un conflit idéologique et religieux. Au motif que la mémoire - telle que les Saturnales l'incarnent ou la résument - a oublié le conflit, l'a passé au filtre d'une mémoire sélective, on dénie au passé sa dimension tragique et conflictuelle. Autrement dit encore, sans jamais vraiment l'avouer - tout en disant même à plusieurs reprises ce que la position d'A. Cameron a de systématique ${ }^{22}-$ le livre de C. W. Hedrick me parait, par le choix même de son angle d'approche, très affadi et dépolitisé, cautionner sans le dire la thèse générale de ce dernier.

Les conséquences de l'appartenance des auteurs à l'une ou l'autre des écoles de pensée dont je viens de présenter de manière sans doute abusivement succincte les positions sont de haute importance. Je ne donnerai ici qu'un exemple, mais qui a de vastes implications. Il s'agit des relations littéraires qui ont pu exister entre saint Jérôme et l'auteur de l'Histoire Auguste. Cette question largement débattue me paraît constituer une manière de symbole de toute la question envisagée ici. Les deux auteurs sont contemporains, c’est indéniable. On a relevé depuis un certain temps que la préface de la Vita Probi de l'Histoire Auguste offrait d'incontestables ressemblances avec la préface de

\footnotetext{
${ }^{21}$ Cf. TROUT, D. Bryn Mawr classical review, 07-11-2001, en ligne.

${ }^{22}$ Par exemple p. 82-83, où il approuve la dimension "nostalgique" des Saturnales pour mieux contrer A. Cameron et insister sur l'idée que "nostalgie” ne va, au fond, pour le dire avec mes propres mots, ni sans “désir de retour” au passé ni sans “douleur”.
} 
la Vita Hilarionis de Jérôme. Or un débat désormais classique s’est engagé pour savoir qui a copié l'autre. ${ }^{23}$ Naturellement le sens de la parodie ou de l'emprunt change du tout au tout selon que c'est l'auteur païen qui pastiche le chrétien ou l'inverse. Dans le premier cas, le fait prend une signification idéologique et religieuse: non seulement un auteur païen avait lu une œuvre d'édification chrétienne - ce que beaucoup répugnent à admettre - mais encore il la tournait en dérision en proposant un empereur romain comme rival dans les sommets de la vertu à un ascète chrétien. Dans le second cas, les choses prêtent moins à contestation. En effet l'interprétation religieuse du procédé - la christianisation de l'héritage historique païen - est mieux attestée, un auteur chrétien qui lit les historiens païens étant chose commune à l'époque et Jérôme étant en outre un familier des breuiaria païens du temps qu'il adaptait, dans sa Chronique, à son projet apologétique personnel. ${ }^{24}$ On assiste donc autour de ce cas d'école que constitue la Vita Probi à un véritable acharnement de contradicteurs qui veulent à tout prix démontrer la véracité de leur thèse. Le dernier développement de l'affaire a été fourni il y a peu par l'échange assez vif qu'ont eu sur le sujet, par publications interposées, N. Adkin ${ }^{25}$ et F. Paschoud, ${ }^{26}$ le second défendant la thèse de la parodie païenne, le premier la thèse de l'antériorité de l'Histoire Auguste. Je crois avoir de mon côté relevé d'assez nombreux emprunts dans la Vita Gallieni de l'Histoire Auguste à la correspondance de Jérôme, notamment à l'epistula 22 à Eustochium et je me range donc du côté de J. Straub, A. Chastagnol, J. Schlumberger et F. Paschoud pour ne citer qu'eux. Quoi qu'il en soit de ma position personnelle sur la question, je souhaitais ici simplement illustrer les crispations des positions autour d’enjeux majeurs pour la compréhension de la période et des relations ou interconnexions entre les œuvres.

\footnotetext{
${ }^{23}$ Bon résumé dans HENGST, D. Den. The pPrefaces in the Historia Augusta. Amsterdam, 1981, p. 119-139; et PASCHOUD, F. Histoire Auguste v. 2. Paris: CUF, 2001, p. 46-49.

${ }^{24} \mathrm{Cf}$. mes études sur deux auteurs chrétiens redevables au païen Eutrope, Les Romana de Jordanès et le Bréviaire d'Eutrope, L'Antiquité Classique 65, 1996, p.175-187; La lecture chrétienne du Bréviaire d'Eutrope (9, 2-5) par Jérôme et Orose, Latomus 56 (2), 1997, p. 264-278.

${ }^{25}$ ADKIN, N. Is the Historia Augusta really indebted to Jerome?, Klio 85 (2), 2003, p. 436-441; N. Adkin reprend la ligne élaborée par A. Cameron, compte rendu de SYME, R. Ammianus and the Historia Augusta, The Journal of Roman Studies 61, 1971, p. 258.

${ }^{26}$ Compte rendu de ADKIN, N. Jerome on Virginity. A commentary on the Libellus de uirginitate seruanda (Letter 22), Cambridge, 2003, Gnomon 77 (7), 2005, p. 594-599.
} 


\section{Rutilius Namatianus et Symmaque}

Avant d'aller plus loin il me semble nécessaire de commencer par réfuter quelques-unes des affirmations inexactes d'A. Cameron.

Dans son étude la plus complète sur les relations entre païens et chrétiens, ce dernier dénie toute existence au cercle de Symmaque, c'est-à-dire que non seulement il conteste l'existence d'une activité intellectuelle concertée dans l'entourage des Symmachi-Nicomachi mais encore il va jusqu'à refuser de reconnaître certains des liens d'amitié qu'ont relevés les historiens entre les satellites qui gravitent autour de ce noyau. C'est ainsi qu'à propos de Rutilius Namatianus, auteur du De reditu suo en 417, il écrit ceci: "As for Rutilius, there is nothing whatever beyond their common paganism to associate him with Symmachus. Not only is he nowhere mentioned in Symmachus' extensive correspondence or included among the interlocutors of Macrobius' Saturnalia; his famous little poem De reditu suo (...) was not written till fifteen years after Symmachus' death. To count him even on the outermost fringes of Symmachus' circle nonetheless, is surely to stretch the concept beyond the point where it has any utility."27

“En ce qui concerne Rutilius Namatianus il n'existe aucune raison - en dehors de leur commun paganisme - de l'associer avec Symmaque. Non seulement il n'est jamais mentionné dans la vaste correspondance de Symmaque mais encore il n'est pas placé parmi les interlocuteurs des Saturnales de Macrobe. Son fameux poème De reditu suo n'était pas encore écrit 15 ans après la mort de Symmaque. L'inclure même aux franges les plus extrêmes du cercle de Symmaque, c'est sans aucun doute étendre le concept au delà de toute utilité”.

Il est parfaitement exact que Rutilius n'est pas un correspondant de Symmaque et non moins vrai que son nom n’est pas cité dans les Saturnales. Mais il est facile de prouver que l'affirmation initiale de Cameron ("il n'y a rien en dehors de leur paganisme commun qui permette de faire le lien entre Rutilius et Symmaque") est, quant à elle, parfaitement fausse. Voici pourquoi.

Dans les vers 542-558 Rutilius se livre à un véritable panégyrique en dimension réduite - il le compare à Cincinnatus et à Régulus - de Protadius, à qui il rend visite au cours d'une étape de son voyage, dans sa résidence à proximité de Pise, en Étrurie. Or Protadius est tout sauf un inconnu: il fut préfet de la ville en 401 et peut-être, à ce poste même, le successeur de Nicomaque Flavien

\footnotetext{
${ }^{27}$ Paganism and Literature... (cité n. 14), p. 4.
} 
Junior. ${ }^{28}$ Et il comptait au nombre des amis de Symmaque à qui l'épistolier adresse les lettres 17 à 34 du livre 4 de la Correspondance: dix-neuf lettres au total, bienveillantes et amicales. Protadius est donc l'ami commun et le proche de Symmaque et de Rutilius: comment dès lors peut-on soutenir qu'il n'existe aucun lien entre les deux hommes? Mais ce n'est pas tout: une autre figure de l'aristocratie de l'époque était elle aussi une relation commune à Rutilius et à Symmaque. En effet le poète Messala, Préfet du Prétoire en 399-400, est cité au vers 268 du livre $1 \mathrm{du}$ De reditu et il est connu de Macrobe. ${ }^{29}$ Symmaque, enfin, lui adresse les lettres 81 à 92 du livre 7 de sa Correspondance. Ne dispose-ton réellement d'aucun élément excepté leur commun paganisme qui permette d'établir un lien entre Rutilius et Symmaque? J'ai la faiblesse de penser que la démonstration du contraire est clairement et définitivement faite.

Quant au lien entre les Saturnales et Rutilius, je l'ai, je crois, clairement établi en 2006 en mettant en évidence plusieurs rapprochements. ${ }^{30}$ Le plus curieux est sans doute la caractérisation identique dans les deux œuvres du dieu Inuus, présenté par Macrobe comme une figure du dieu Pan et un avatar du soleil, ${ }^{31}$ ce qui est exactement déjà le cas dans le De reditu. ${ }^{32}$ Enfin, il existe entre Servius et le De Reditu des relations qui font penser que Rutilius a lu le grammairien et lui a emprunté trois explications étymologiques au moins. ${ }^{33}$

Je ne veux pas m'étendre outre mesure sur le sujet. En mettant un certain nombre d'éléments au jour et en faisant ces rappels salutaires je voudrais simplement souligner combien il est aisé en dissimulant une partie du dossier de fausser la perception et l'appréciation des choses. De fortes connexions existent entre le cercle des amis de Symmaque et celui de Rutilius Namatianus, des emprunts de ce dernier à Servius peuvent être relevés, de très nombreux points de contacts idéologiques, enfin, se révèlent entre Servius et l'Histoire Auguste.

\footnotetext{
${ }^{28}$ PLRE I, p. 751-752; pour une discussion sur l'ordre de succession des préfets de la Ville entre 400 et 402, cf. CHASTAGNOL, A. Les fastes de la préfecture de Rome au Bas-Empire. Paris, 1962, p. 253-260 et CALLU, J.-P. Symmaque, lettres, livres VI-VIII. Paris: CUF, 1995, p. 179, note 67. ${ }^{29}$ Sat. 1, 6, 26.

${ }^{30}$ Rutilius Namatianus, Aelius Aristide et les chrétiens, Antiquité Tardive 14, 2006, p. 235-244, ici p. 241-243 et note 82 .

${ }^{31}$ Sat. 1, 22, 2-4.

${ }^{32}$ De reditu 1, 232: cf. mon étude Rutilius Namatianus... (cité n. 30), p. 241 et note 80.

${ }^{33}$ Rutilius Namatianus... (cité n. 30), p. 241.
} 


\section{L'Histoire Auguste et Nicomaque Flavien senior}

L'un des arguments que l'on entend le plus souvent répété par les opposants à la vision polémique des relations pagano-chrétiennes consiste à présenter comme une vérité établie le fait que les païens ne lisaient pas les œuvres chrétiennes alors que l'inverse n'est pas vrai, ${ }^{34}$ Les chrétiens devraient ainsi leur victoire au fait qu'ils ont su tirer profit de leurs lectures païennes pour retourner contre leurs auteurs leurs arguments mêmes, tirés de leurs propres autorités. Cette thèse est d'ailleurs celle-la même qu’ont développée les chrétiens dès l'Antiquité Tardive et qu'expose fort clairement l'histo-

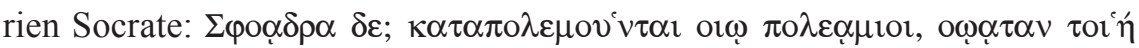

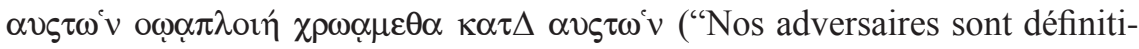
vement vaincus lorsque nous retournons contre eux leurs propres armes"). ${ }^{35}$

La seconde partie de l'affirmation - les chrétiens lisaient les païens - ne fait aucun doute: Jérôme, Orose et d'autres ont su christianiser avec une habileté non dénuée de mauvaise foi les historiens païens, de Tite-Live à Eutrope. Quant à la première partie de l'affirmation - les païens ne lisaient pas les chrétiens - je crois qu'elle est dans sa généralisation abusive très largement fausse. Je rappellerai au passage que la première réfutation en règle du christianisme qui nous soit connue, à savoir l'ouvrage de Celse contre les Chrétiens - le Logos Alethès ou Discours Vrai, dans le dernier quart du second siècle, dont Origène nous a préservé de très larges fragments - repose sur une lecture minutieuse et de première main de certaines parties de la Bible. P. de Labriolle a ainsi rappelé que Celse connaissait partiellement l'Ancien Testament, l'Évangile de saint Matthieu et sans doute celui de saint Luc. ${ }^{36}$ De leur côté J. Fontaine et Y.-M. Duval pensaient que si les preuves étaient difficiles à réunir en raison du petit nombre de recherches sur le sujet et aussi du fait de la rareté des sources il était hautement vraisemblable que les païens du quatrième siècle connaissaient la poésie chrétienne contemporaine. ${ }^{37}$ Y.-M. Duval estime par exemple que Paulin

\footnotetext{
${ }^{34}$ CAMERON, A. Paganism and Literature... (cité n. 14), p. 8-10; WOLFF, É., LANCEL, S., SOLER, J. Rutilius Namatianus. Sur son retour. Paris: CUF, 2007, p. XV.

${ }^{35}$ SOCRATE 3, 16, 18, p. 211 Hansen.

${ }^{36}$ La réaction païenne (cité n. 11), p. 125-126. Peut-être avait-il lu d'autres parties encore de la Bible. Sa connaissance de la littérature apologétique du second siècle fait en revanche débat: cf., ibid.,p. 126.

${ }^{37}$ Christianisme et formes littéraires (cité n. 14), p. 412-413 (discussion qui suit l'exposé de HERZOG, R. Probleme der Heidnisch-Christlichen Gattungskontinuität am Beispiel des Paulinus von Nola, ibid., p. 373-411).
} 
de Nole était resté après sa conversion en contact avec l'aristocratie païenne et que son Carmen 22 à Jovius n'était pas destiné à être lu par son seul dédicataire chrétien. ${ }^{38}$ Augustin, enfin, nous apprend que le rhéteur païen Marius Victorinus lisait non seulement l’Écriture sainte mais toute la littérature chrétienne. ${ }^{39}$

De mon côté j'ai exposé ailleurs les éléments concordants qui me font penser que le De reditu de Rutilius détourne sciemment un certain nombre de formules chrétiennes devenues en son temps déjà fameuses pour alimenter une polémique de nature idéologique et religieuse avec les chrétiens: je pense notamment à l'accusation lancée contre le judaïsme, radix stultitiae, ${ }^{40}$ au tour parodique numerare stellas ou encore au thème plus général de la regeneratio ou ratio renascendi, un motif qui cherche à rivaliser avec celui de la résurrection chrétienne. ${ }^{41}$ Il n'est pas jusqu'à la poésie de Claudien - je pense l'avoir démontré récemment ${ }^{42}$ - qui ne contienne, ici ou là, par exemple dans l'In Rufinum, d'assez transparentes piques contre le christianisme dont tout le sel repose dans la savante adaptation de formules bibliques.

Et puis il me faut bien revenir sur l'Histoire Auguste. A. Cameron avait tenté, en quelques lignes de son article de 1966, de balayer d'un revers de main l'interprétation circonstanciée de J. Straub ${ }^{43}$ qui, l'un des premiers, avait mis en évidence le discours antichrétien que véhiculait cette collection de biographies: "Thus it is a more than ordinarily damaging blow to this view as a whole that the Historia Augusta so obviously does not have any clear cut religious point of view". ${ }^{44}$ Passons sur la rapidité de l'exécution pour ne retenir qu'un argument (le seul) de Cameron: l'œuvre est anonyme et ne peut donc être interprétée, compte tenu de l'ignorance où nous sommes de son auteur et de sa date de rédaction. Or, précisément, l'Histoire Auguste n’est plus une œuvre anonyme

\footnotetext{
${ }^{38}$ Christianisme et formes littéraires (cité n. 14), p. 413.

${ }^{39}$ Conf. 8, 2, 4: legebat, sicut ait Simplicianus, sanctam scripturam omnesque christianas litteras inuestigabat studiosissime et perscrutabatur.

${ }^{40}$ Cf. GUILLAUMIN, J.-Y. Radix stultitiae chez Rutilius Namatianus (vers 389), Vita Latina 174, 2006, p. 114-122.

${ }^{41}$ Rutilius Namatianus... (cité n. 30), p. 240-241.

${ }^{42} \mathrm{Cf}$. Une lecture religieuse des invectives de Claudien est-elle possible?, Antiquité Tardive 16, 2008, L'Empire des Théodoses, p. 177-186 et Antiquus error (cité n. 3), Partie V, chapitre 1.

${ }^{43}$ CAMERON, A. Paganism and Literature... (cité n. 14), p. 9, note 2, renvoie de manière elliptique à un compte rendu paru dans The Journal of Roman Studies 55, 1965, p. 240; mais, bien sûr, l'ouvrage majeur de J. Straub est Heidnische Geschichtsapologetik in der christlichen Spätantike. Untersuchungen über Zeit und Tendenz der Historia Augusta. Bonn, 1963.

44 "Paganism and Literature..." (cité n. 14), p. 10.
} 
et le contexte de sa rédaction se précise nettement. On me pardonnera de citer mes propres travaux, mais comment faire autrement en l'occurrence?

Le débat sur l'auteur de l'Histoire Auguste a précisément connu, au printemps 2005, lors du colloque Histoire Auguste de Bamberg, un rebondissement spectaculaire dont je suis responsable. Les Actes de ce colloque étant aujourd'hui sous presse, je me permets d'exposer ici, de manière résumée et simplifiée, la démonstration que j'ai présentée alors. ${ }^{45}$. L'identification de l'auteur que je propose met un terme, je crois, à l'énigme de l'Histoire Auguste. Mon point de départ est une évidence oubliée: le genre biographique et le genre annalistique, artificiellement distingués par une très vieille tradition scolaire, ne font en réalité qu'un. D'ailleurs les auteurs anciens ne distinguaient pas les Histoires et les Annales de Tacite, ce sont les éditeurs modernes qui opèrent ce distinguo qui n'a pas lieu d'être. Saint Jérôme, en outre, parle à propos de l'œuvre de Tacite, de biographies d'empereurs (uitae Caesarum): "Cornélius Tacite, lui aussi, qui, depuis la mort d'Auguste jusqu'à la mort de Domitien a rédigé les Vies des Césars en trente livres” (Cornelius quoque Tacitus, qui post Augustum usque ad mortem Domitiani uitas Caesarum triginta uoluminibus exarauit). ${ }^{46}$ Jérôme ne fait, en l'état, qu'exprimer la perception que les Anciens avaient de ces œuvres: ils y voyaient bien un recueil de Vitae. Le titre que donne à l'Histoire Auguste le plus ancien manuscrit connu, le Codex Palatinus Latinus 899, daté du IX ${ }^{\mathrm{e}}$ siècle et conservé à la Vaticane, titre diversement mais assez fidèlement reproduit par d'autres manuscrits postérieurs, Vitae diuersorum principum et tyrannorum a diuo Hadriano usque ad Numerianum, "Vies des divers princes et tyrans du divin Hadrien à Numérien”, n’annonce donc en rien un contenu fondamentalement différent de ce que l'on lisait chez Tacite.

Ce point établi, on doit relire de près un passage jusque-là négligé de la Vie d'Aurélien dans lequel l'auteur de l'Histoire Auguste se présente comme l'auteur d'annales. Il figure dans la Vita Aureliani. Le biographe veut prouver que le futur empereur avait acquis sous le règne de Claude une réelle réputation qui le destinait un jour à assumer l’Empire. ${ }^{47}$ Pour prouver la véracité de ses dires, il s’apprête à citer une lettre de Claude à Aurélien. C’est la formule introductive

\footnotetext{
${ }^{45}$ RATTI, St. Nicomaque Flavien senior auteur de l'Histoire Auguste, dans BONAMANTE,G. et BRANDT, H (éd.) Historiae Augustae Colloquium Bambergense Bari, 2007, p. 305317. On trouvera dans cette étude la bibliographie dont je décharge ici mon résumé. ${ }^{46}$ In Zach. 3, 14.

${ }^{47}$ Aurelian. 16, 1.
} 
qui m'intéresse ici: “Il subsiste une lettre que, pour ma part, selon mon habitude, par souci d'authenticité, ou plutôt selon la pratique que, je le vois, ont suivie les autres auteurs d'annales, j'ai jugé nécessaire de citer" (Exstat epistula quam ego, ut soleo, fidei causa, immo ut alios annalium scriptores fecisse uideo, inserendam putaui). ${ }^{48}$ Il est donc clair que l'Histoire Auguste n'appartient pas aux yeux de son propre auteur au genre biographique mais qu'elle relève du genre des annales. Cette précision constitue ma deuxième conclusion et elle ouvre de grandes perspectives.

Nous connaissons, en effet, grâce à une inscription datée de 431, rédigée sur la base d'une statue à son effigie, l'existence d'une œuvre historique d'importance rédigée par Nicomaque Flavien senior. ${ }^{49}$ L'inscription appelle cette œuvre des Annales et nous apprend que Théodose avait souhaité que cette œuvre lui fût dédicacée. Nous ne possédons malheureusement aucun fragment de cet ouvrage dont l'existence ne fait néanmoins aucun doute. Nicomaque Flavien senior n'est pas un inconnu. C'était un lettré. Il était le préfet du prétoire de l'empereur Théodose. Son rôle fut considérable dans la lutte entre païens et chrétiens à la fin du quatrième siècle. Nicomaque Flavien fait même figure de chef du camp païen qui, rangé sous la bannière de l’usurpateur Eugène - un chrétien modéré-, s'opposa aux troupes de Théodose lors de la fameuse bataille de la Rivière Froide en 394. Voyant son camp vaincu, Nicomaque Flavien préfère le suicide à la défaite.

La solution est très simple - comme l'est toujours la vérité: Nicomaque Flavien senior, auteur d'Annales, est en réalité l'auteur de l'Histoire Auguste, qui sont, on l'a vu, des annales. J'en trouve la preuve définitive dans le catalogue médiéval de l'abbaye de Murbach, en Alsace. Ce catalogue contient en effet une notice oubliée qui fournit un indice sûr pour considérer que l'Histoire Auguste avait été rédigée en sept livres. Or un autre témoignage - un texte important de Cassiodore, appelé Ordo generis Cassiodororum ou encore Anecdoton Holderi du nom de son inventeur au milieu du $19^{\mathrm{e}}$ siècle - nous apprend que Symmaque le Jeune, qui n'était autre que l'arrière-petit-fils de Nicomaque Flavien senior par sa grand-mère paternelle, avait rédigé, sur le modèle de son ancêtre précise le texte, une histoire en sept livres. Il faut donc identifier l'Histoire Auguste et

\footnotetext{
${ }^{48}$ Aurelian. 17, 1.

${ }^{49}$ CIL 6, 1783 (ILS 2948 Dessau): cuius (scil. Theodosii) in eum (scil. Flauianum) effusa beniuolentia et usque ad annalium quos consecrari sibi a quaestore et praefecto suo uoluit...
} 
les Annales de Nicomaque Flavien senior - qui est en fin de compte l'auteur désormais démasqué du premier ouvrage.

Je crois donc avoir démontré que l'Histoire Auguste a été rédigée, dans son dernier état, entre 392 et 394 et que son auteur n'est autre que Nicomaque Flavien senior, le partisan de l'usurpateur Eugène et le chef de file du camp païen au Frigidus. On démontrera peut-être dans les années à venir que j'ai tort. Pour le moment je n'ai connaissance d'aucun argument valide contre ma découverte, quand bien même je devine sans peine le scepticisme de certains. Il est évident qu'une telle identification entraîne des conséquences de poids quant à l'interprétation religieuse de l'Histoire Auguste. Je ne prétendrai pas ici que ces biographies ne sont qu'une Historia aduersus christianos et la lecture de J. Straub avait quelque chose d'excessif, d'autant que la datation qu'il propose pour l'œuvre (après 416) n'est pas défendable. Certains passages sont en effet d'interprétation délicate et peuvent se lire comme un appel à la tolérance. Néanmoins ma thèse conforte les positions de ceux qui ont cru repérer dans l'œuvre des allusions sous forme de pastiche à la Bible ou aux auteurs chrétiens. ${ }^{50} \mathrm{~J}$ 'ai moimême suggéré, en 2002, que l'auteur de l'Histoire Auguste avait lu Tertullien et Lactance et leur avait répondu sur le mode polémique et humoristique. ${ }^{51}$ La figure d'Apollonios de Tyane qui ressuscite les morts ${ }^{52}$ entre en concurrence avec celle du Christ. Les interactions avec Jérôme elles-mêmes, à l'existence desquelles je crois personnellement, prennent dans ce contexte une nouvelle portée. Il faut donc bien, alors que la science progresse et propose de nouvelles hypothèses, opérer un certain nombre de révisions qui peuvent parfois être déchirantes.

Ce qui précède et que j'ai tenté d'exposer avec le moins de longueurs possibles m'amène à situer ma question initiale sous un horizon nouveau et cette fois dégagé. Comment interpréter les rééditions des œuvres classiques dans le dernier quart du quatrième siècle? Prenons un exemple: les déclamations du pseudoQuintilien. Ce recueil d'exercices d'école sur des sujets divers et d'apparence parfois frivole a probablement été constitué autour de l'année 384, les travaux

\footnotetext{
${ }^{50}$ Cf., en dernier lieu, F. MUNDT, Die Maske des Christen: Spuren christlicher Literatur in der Historia Augusta, dans THOME, G. et HOLZHAUSEN, J. (éd.). Es hat sich viel ereignet, Gutes wie Böses. Lateinische Geschichtssreibung der Spät und Nachantike. München-Leipzig, 2001, p. 37-56. ${ }^{51}$ Réponses de l'Histoire Auguste aux apologistes Tertullien et Lactance, Museum Helveticum 59, 2002, p. 229-237.

${ }^{52}$ Histoire Auguste, Aurelian. 24, 8: ille mortuis reddidit uitam.
} 
récents de C. Schneider l'ont démontré. ${ }^{53}$ J'ai pu de mon côté confirmer cette datation par un élément nouveau. ${ }^{54}$ La troisième des Grandes déclamations a pour sous-titre le Miles Marianus. Il s'agit d'une plaidoirie fictive, cen sée être prononcée par l'avocat d'un soldat de l'armée du grand Marius dénommé Trebonius. Ce dernier, en 104 avant notre ère, au cours de la campagne du général Romain contre les Cimbres, a été victime d'une tentative de viol de la part du propre neveu de Marius, son tribun, Caius Lusius. ${ }^{55}$ Je suis revenu à plusieurs reprises sur la date et l'auteur de ce texte. ${ }^{56}$ Ce qui était acquis jusqu'à présent par les suscriptions des manuscrits des declamationes c'est que Domitius Dracontius avait été, avec Hiérius, l'un des maîtres d'œuvres de l'édition du recueil. ${ }^{57}$ Or Domitius Dracontius est un proche de Nicomaque Flavien senior, la chose est bien établie depuis Léon Hermann ${ }^{58}$ et a été souvent réaffirmée depuis. ${ }^{59} \mathrm{Ce}$ que j'ai démontré, je crois, avec un nombre respectable d'arguments, c'est que Nicomaque Flavien senior ne s'est pas contenté de diriger une opération de réédition, mais qu'il est lui-même l'auteur du Miles Marianus.

\section{Conclusion}

Il est donc clair que si Nicomaque Flavien senior est bien l'auteur de l'Histoire Auguste et du Miles Marianus, non seulement, je l'ai déjà dit, le recueil des biographies impériales prend un sens nouveau mais qu'encore la réédition des declamationes en recueil s'inscrit par la force des choses dans un contexte précis et incontestable, à savoir la lutte idéologique menée contre les chrétiens.

\footnotetext{
${ }_{53}^{53}$ Quelques réflexions... (cité n. 10).

${ }^{54}$ Sur l'auteur et la date du Miles Marianus (Ps. Quint., decl. 3), Antiquus error (cité n. 3), partie IV, chapitre 10.

${ }^{55}$ Cf. SCHNEIDER, C. [Quintilien], Le soldat de Marius (Grandes déclamations, 3): Cassino, Edizioni dell'Università degli Studi di Cassino, 2004, et ma recension critique, Antiquité Tardive 15, 2007, p. 405-408.

${ }^{56}$ Nicomaque Flavien senior et l'Histoire Auguste: la découverte de nouveaux liens, Revue des Études latines 85, 2007, p. 204-219 et Sur l'auteur... (cité n. 54).

${ }^{57}$ Subscriptio à la decl. 11, p. 219 Hakanson: Legi et emendaui ego Dracontius cum fratre Hierio incomparabili grammatico urbis Romae et suscriptio à la decl. 18, p. 371 Hakanson: descripsi et emendaui Domitius Dracontius de codice fratris Hieri.

${ }^{58}$ Hierius et Domitius, Latomus 13, 1954, p. 37-39.

${ }^{59}$ Hiérius est probablement le dédicataire du livre 1 du De pulchro et apto de saint Augustin (vers 380, avant la conversion) et le personnage visé au vers 47 du Carmen contra paganos: cf. MAZZARINO, A. Frater Hierius, Helikon 15-16, 1975-1976, p. 461-464; PLRE I, p. 431; SCHNEIDER, C. Quelques réflexions... (cité n. 10), p. 628-629. Pour Domitius Dracontius, cf. HERRMANN, L. Hierius et Domitius (cité n. 58), p. 39.
} 
Je n'ai eu aucune peine à montrer quel sens le Miles Marianus prenait alors. Après avoir mis en lumière les convergences idéologiques et axiologiques qui existent entre l'Histoire Auguste et la declamatio 3, j'ai suggéré que leur auteur commun cherchait à ne point abandonner aux chrétiens la défense de certaines valeurs telles que la pudicitia ou la sanctitas ou encore à revendiquer en faveur de la tradition païenne la condamnation de certaines pratiques telle que l'homosexualité masculine ${ }^{60}$ Cette interprétation illustre par conséquent combien les rééditions d'œuvres classiques entre 384 et 408 n'avaient pas pour objectif premier l'établissement d'un texte sûr de l'auteur en question. ${ }^{61}$ Elles étaient, au fond, marquées par le contexte dans lequel elles voyaient le jour, ce qui conforte considérablement l'interprétation polémique au détriment de la vision irénique des relations pagano-chrétiennes. On voit bien ainsi comment la diffusion des convictions néoplatoniciennes d'Apulée telles qu'elles sont affichées dans l'Apologie ou encore le nationalisme livien pouvaient contribuer à servir la cause des nostalgiques des temps anciens.

Quant à la poésie satirique, illustrée par Martial, Perse et Juvénal, la faveur dont elle jouit auprès des intellectuels païens de la fin du quatrième siècle doit être mise en relation avec les emprunts que les chrétiens faisaient depuis peu à ce genre qui puise ses racines dans le vieux fonds romain. On lit en effet dans le triptyque antipaïen anonyme formé par le Carmen contra paganos, le Carmen ultimum et le Carmen ad senatorem de nombreux vers, gorgés de venin, dirigés contre les pratiques païennes abhorrées, que l'on pourrait croire dans leur forme empruntés à Horace ou à Juvénal. ${ }^{62}$ Les païens ne demeurent pas en reste, usent en faveur de leur camp de la verve satirique et ce faisant n'entendent pas abandonner aux chrétiens l'une des plus anciennes spécificités de leur littérature nationale. L'éloge que Rutilius Namatianus, quelques années plus tard, fera du poète satirique Lucillus, un aristocrate de ses amis (il fut quelques

\footnotetext{
${ }^{60}$ On ajoutera que la portée idéologique et polémique antichrétienne de la declamatio 10 du pseudoQuintilien (le Sepulcrum incantatum) a été parfaitement mise en évidence par SCHNEIDER, C. et URLACHER, C. Rationnel et irrationnel dans le Sepulcrum incantatum du pseudo-Quintilien. Les enjeux d'une dialectique, dans Mirabilia - Conceptions et représentations de l'extraordinaire dans le monde antique. éd. BIANCHI, O. et THÉVENAZ, O. sous la dir. de Ph. Mudry, Bern, 2004, p. 99-113. ${ }^{61}$ Je rejoins sur ce point ce que J. E. G. ZETZEL a écrit à propos des révisions de Tite-Live et de Fronton: cf. The subscriptiones in the manuscripts of Livy and Fronto and the meaning of emendatio, Classical Philology 75, 1980, p. 38-59.

${ }^{62} \mathrm{Cf}$. Le consul de $382 \mathrm{Fl}$. Claudius Antonius fut-il un auteur antipaïen?, Revue des Études Latines 60, 1982, p. 298-312, ici p. 303.
} 
années avant 417 comes sacrarum largitionum). ${ }^{63}$ "dont la muse assassine ne le cède ni à Turnus ni à Juvénal", ${ }^{64}$ n'atteste pas seulement le retour en grâce d'un genre mais démontre que le recours à la lima censoria participe du combat pour le rétablissement des valeurs morales antiques. Restituit ueterem censoria lima pudorem $^{65}$ dit l'auteur du De reditu à propos de Lucillus; c'est exactement ce que je dirais pour ma part de l'entreprise de réédition des œuvres classiques à la fin du quatrième siècle: partiellement collective et en partie concertée, elle a été pensée au service d'un idéal païen comme une tentative de restitutio rerum in pristinum statum.

\footnotetext{
${ }^{63}$ De reditu suo 607.

${ }^{64}$ De reditu suo 603-604: Huius uulnificis satira ludente Camenis / Nec Turnus potior nec Iuuenalis erit.

${ }^{65}$ De reditu suo 605.
} 